\title{
Observation of Diamagnetic Domains in Beryllium by Muon Spin Rotation Spectroscopy
}

\author{
G. Solt, ${ }^{1}$ C. Baines, ${ }^{1}$ V. S. Egorov,${ }^{2}$ D. Herlach, ${ }^{1}$ E. Krasnoperov, ${ }^{2}$ and U. Zimmermann ${ }^{1}$ \\ ${ }^{1}$ Paul Scherrer Institut, $\mathrm{CH}-5232$ Villigen PSI, Switzerland \\ ${ }^{2}$ Russian Research Center, Kurchatov Institute, Moscow 123182, Russia
}

(Received 29 November 1995)

\begin{abstract}
Spectroscopic evidence is given for the recurrent "domain phase" with oppositely magnetized regions in diamagnetic beryllium. A periodic splitting of the precession frequency of implanted muons in a Be single crystal was observed as the strength of the applied field $\mathbf{H} \|\langle 0001\rangle$ varied near $H_{0}=2.74 \mathrm{~T}$. On sweeping $H$ downwards, the doublet emerges and persists within more than one third of each de Haasvan Alphen cycle, the positions of the lines fixed, with the lower frequency (diamagnetic) component gaining and the higher (paramagnetic) one losing intensity. The line splitting gives $\Delta B \approx 29 \mathrm{G}$ for the induction difference in domains at $T=0.8 \mathrm{~K}$.
\end{abstract}

PACS numbers: 75.20.En, 75.60.Ch, 76.75.+i

The periodic formation and disappearance of a phase with diamagnetic and paramagnetic domains in a normally diamagnetic metal in strong magnetic fields at low temperatures was predicted by Condon [1]. The domains arise because of the strong magnetic interaction of electrons on quantized Landau orbitals in metals, for which the de Haas-van Alphen (dHvA) oscillation of the orbital susceptibility has a sufficiently large amplitude $\kappa_{0}[1,2]$. For a pure single crystal at low temperature, $\kappa_{0}$ is determined by the parameters of the Fermi surface (extremal area of the cross section normal to $\mathbf{H}$, the variation of this area along the field direction, cyclotron mass) and by the field strength $H$. When, for a given range of $H$, one has

$$
\kappa_{0}=|\partial M / \partial B|_{\max }>1 / 4 \pi,
$$

this implies $\partial H / \partial B=1-4 \pi \kappa<0$ within some part of each dHvA oscillation period, indicating thermodynamical instability and the multivaluedness of the induction $B(H)$. For a long rodlike sample oriented along $\mathbf{H}$, the instability is avoided by $B$ jumping between two stable values $B_{1}, B_{2}$ at a given $H=H_{c}$, leaving out all $B$ values in the discontinuity interval $\Delta B=\left(B_{1}, B_{2}\right)$, which do not occur in the sample [3]. The consequence for a platelike sample in perpendicular field is that $B$ cannot "follow" the continuously varying field $H$ through the forbidden intervals, as the boundary condition $B=H$ for a uniformly magnetized state at this geometry would require. For $H$ in the range $B_{1}<H<B_{2}$, the magnetic state is thus predicted [1] to break into alternating regions of diamagnetic and paramagnetic magnetization, with inductions $B=B_{1}$ and $B=B_{2}$, respectively, the proportion of the domains varying with $H$ so that $\bar{B}=H$ is fulfilled as an average over the sample. The $H-B$ diagram is similar to the $p-v$ diagram of a van der Waals gas, only with more than one discontinuity interval $\Delta B$, situated periodically on the $B$ axis. A series of phase transitions are therefore expected, the phase of paramagnetic and diamagnetic domains with boundaries parallel to $\mathbf{H}$ returning and disappearing within each $\mathrm{dHvA}$ period of length $\Delta H=B_{0}^{2} / F$. Here $F$ is the "dHvA frequency" in terms of which $\kappa$ is given by [2]

$$
\begin{aligned}
\kappa(B) & =\kappa_{0}(B) \cos (2 \pi F / B+\phi) \\
& \approx \kappa_{0}\left(B_{0}\right) \cos \left[2 \pi\left(B-B_{0}\right) / \Delta H\right]
\end{aligned}
$$

[the second expression holds for $\Delta H \ll B_{0}$ within a few cycles near $B_{0}$, chosen so that $\left.\kappa\left(B_{0}\right)=\kappa_{0}\right]$. The state with domains ("dHvA phase") is physically similar to the intermediate state of type I superconductors, reconciling apparently conflicting requirements of the electronic ground state and electrodynamical boundary conditions by forming a two-phase system.

The existence of the dHvA phase has been experimentally confirmed, though only in the single example of silver [4]. The domains were revealed by a periodic splitting of the NMR line, corresponding to the difference $\Delta B \approx 12 \mathrm{G}$ in the paramagnetic and diamagnetic regions in the field of $H_{0}=9 \mathrm{~T}$ [4] at $4.2 \mathrm{~K}$. In spite of intensive research since 1966, no spectroscopic evidence for dHvA domains in any other metals could be obtained, and a detailed experimental study of the structure is still lacking. The efforts have been concentrated mainly on beryllium, for which Eq. (1) is fulfilled at the relatively low fields of $H_{0} \sim 2-3 \mathrm{~T}$, and the oscillations of several macroscopic quantities (susceptibility, magnetoresistance, thermopower) have given strong, though indirect support to the predicted dHvA phase [2,57]. The NMR experiment was attempted, but "the inherent nuclear quadrupole splitting made the data collection and interpretation difficult" [4], and although a "periodic line broadening was observed, its explanation in terms of a $\Delta B$ splitting was not clear."

As also noticed earlier [8], the technique of muon spin rotation $(\mu \mathrm{SR})$ eliminates the difficulty of quadrupolar broadening $\left(S_{\mu}=1 / 2\right)$ and is, thereby, a priori the ideal method to study the dHvA domain phase. However, the spread in frequency of the precessing muons depends on the localization and dynamics of the $\mu^{+}$in the given crystal at low temperatures, and it has to be checked first if this linewidth allows the desired frequency resolution. This proved to be the case for our Be single crystal sample. 
We report here on the first spectroscopic evidence for recurrent diamagnetic and paramagnetic $\mathrm{dHvA}$ domains in beryllium by using $\mu \mathrm{SR}$. The muons, thermalized randomly in the diamagnetic and paramagnetic regions, give rise to two distinct $\mu^{+}$-spin precession frequencies. The linewidth in the absence of a domain phase below $T=4 \mathrm{~K}$ was seen to correspond to $\$ 2 \mathrm{G}$ in local field fluctuations, whereas the expected difference in induction $\Delta B$ between diamagnetic and paramagnetic domains should be an appreciable fraction of $\Delta H \approx$ $80 \mathrm{G}$ near $H_{0}=2.7 \mathrm{~T}$. A great advantage of $\mu \mathrm{SR}$ is that the entire volume of a metal single crystal can be studied, and not only that within a skin depth. We used a plate of $1 \times 1 \times 0.2 \mathrm{~cm}^{3}$ which was sufficiently thick for both complete muon absorption and insuring a negligible influence of the predicted domain distortion at the surface. The mean penetration length of the $\mu^{+}$ beam (of momentum $\approx 28 \mathrm{MeV} / c$ ) is $\sim 0.6 \mathrm{~mm}$ in $\mathrm{Be}$, much more than the depth $d$ of the surface layer where the domains "open." [One has $d \sim w$, where $w$ is the domain width, and the estimate $w \sim(Z D)^{1 / 2}$ [2], with sample thickness $Z=0.2 \mathrm{~cm}$ and electron orbit diameter $D \approx 10^{-4} \mathrm{~cm}$, gives $w \approx 0.04 \mathrm{~mm}$.]

The experiment was performed at the $\mu$ SR facility of the Paul-Scherrer-Institut, Villigen; for a description of the $\mu \mathrm{SR}$ method, see, e.g., Ref. [9]. The beam of low energy $(4 \mathrm{MeV}) \mu^{+}$particles, with a spin polarization $\mathbf{P}_{0}$ rotated by an angle of $\approx 50^{\circ}$ "out" of the beam direction, was directed perpendicularly at the single crystal plate, cut normal to the $c$ axis; the applied field $\mathbf{H}$ was parallel to beam and $c$ axis.

The frequency spectrum of the polarization $\mathbf{P}(t)$ precessing about $\mathbf{H}$ was analyzed on varying $H$, by steps of $7 \mathrm{G}$, near $H_{0}=2.7 \mathrm{~T}$. The homogeneity of the applied field at the sample was tested at the onset by cooling the cryostat down to $0.8 \mathrm{~K}$ and in situ measuring the oscillations of the thermopower $U(H)$ at two field intervals near $\mathbf{H}_{0}=2.7 \mathrm{~T}$. The precession of $\mathbf{P}$ is measured by counting the (oscillating) number of decay positrons in detectors perpendicular to the beam direction [9]. For beryllium in the normal, homogeneous phase at helium temperatures, the oscillating intensity $I$ [after separation of the trivial factor $\exp \left(-t / \tau_{\mu}\right)$ related to the $\mu^{+}$lifetime $\tau_{\mu}$ ] was seen to be well described by

$$
I(t)=I_{0} e^{-\lambda t} \cos \left(\gamma_{\mu} B t+\delta\right),
$$

where $\gamma_{\mu}=2 \pi \times 13.554 \mathrm{kHz} / \mathrm{G}$ is the gyromagnetic ratio for $\mu^{+}$. In the absence of domains $\lambda \approx 10^{5} \mathrm{~s}^{-1}$, corresponding to the quoted value $\approx 1-2 \mathrm{G}$ of random dipolar fields.

A first analysis of the results, obtained by tuning $H$ about $H_{0}=2.74 \mathrm{~T}$ at $T=0.8 \mathrm{~K}$, was done in the "broadening model" on fitting $A(t)$ to the data by Eq. (1). Since the nuclear spins are randomly oriented along $\mathbf{H}$ at these field strengths and temperatures, any deviation from $\lambda(H) \approx$ const must reflect the influence of electron orbital magnetism. The dependence of $\lambda$ on $H$ is shown in Fig. 1. The expected $\lambda \approx 10^{5} \mathrm{~s}^{-1}$ values appear only exceptionally near the minima, the function $\lambda(H)$ rises, reaches values about 10 times as large as at minimum, and descends again, with a period of $76 \pm 2 \mathrm{G}$ coinciding, within this accuracy with the dHvA frequency $\Delta H=$ $78 \mathrm{G}$ at $H_{0}=27400 \mathrm{G}$. (In the figures the nominal field values $H$ are replaced by the real ones, deduced from the $\mu^{+}$precession frequencies outside the splitting region; the accuracy in $H$ is $\approx \pm 1 \mathrm{G}$.)

Although the broadening model is, strictly speaking, adequate only for Lorentzian broadening (i.e., near the minima in Fig. 1), and the "broadened line" in our case is a well resolved doublet for $\lambda \approx 0.4 \mu \mathrm{s}^{-1}$ or larger, the maximum of $\lambda \approx 0.9 \mu \mathrm{s}^{-1}$ gives a qualitatively correct idea of the splitting, $\Delta \nu \approx \lambda / \pi \approx 0.3 \mathrm{MHz}$, equivalent to $\Delta B \approx \Delta \nu /\left(\gamma_{\mu} / 2 \pi\right)=22 \mathrm{G}$. The exact value found below is $\approx 29 \mathrm{G}$, and this means that the interval $\delta H$, where the domain phase exists, is more than one-third of the dHvA period $\Delta H$ at this $H_{0}$.

Three raw frequency spectra, taken at the central peak region in Fig. 1, are shown in Fig. 2. The applied field $H$ for the upper spectrum is somewhat higher than $H_{m}$, at which the maximum of $\lambda$ occurs, the spectrum in the middle is taken just at $H_{m}$, and the lower spectrum is for $H<H_{m}$. The stable position of the doublet lines, as $H$ sweeps downwards, and the "transfer" of intensity into the lower frequency line, are spectacular and unambiguous evidence for the presence of domains and their varying volume ratio following the variation of $H$.

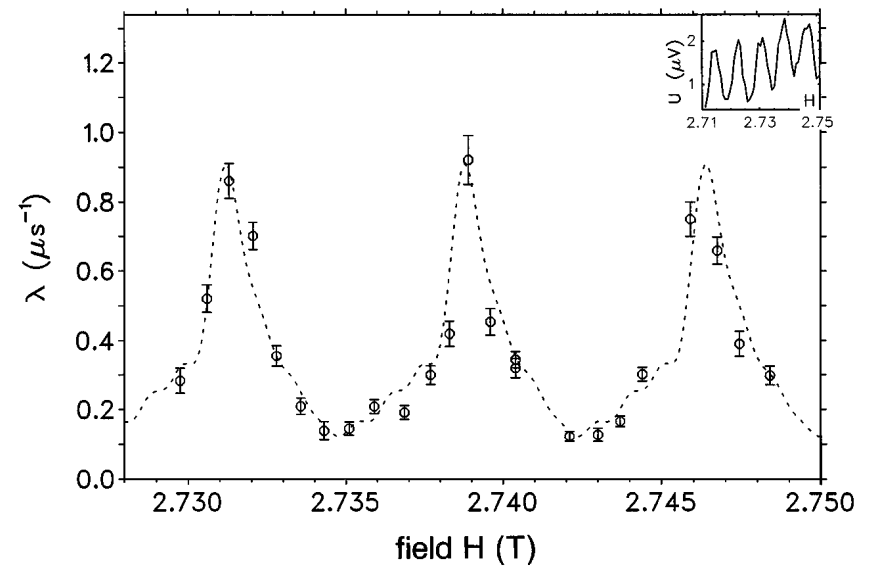

FIG. 1. Exponential damping rate $\lambda$ of the precessing $\mu^{+}$ polarization, as a function of $H$. The periodic sharp rise of $\lambda$ (i.e., of the width $\Delta \omega=2 \lambda$ of the spectral line in frequency space at $\omega=\gamma_{\mu} B$ ) marks the onset of line splitting due to the incipient domain phase. Above $\lambda \approx 0.4 \mu \mathrm{s}^{-1}$ the "broadened" line is, in fact, a well resolved doublet (Fig. 2). The dotted line is a best fit to the data by a (truncated) Fourier series with a period of $\Delta H=76 \mathrm{G}$. This period agrees, within the present accuracy, with the value of the dHvA period $\Delta H=78.2 \mathrm{G}$ at $H_{0}=2.74 \mathrm{~T}$. The in situ measured oscillations of the thermopower are shown in the inset. In all figures, $T=0.8 \mathrm{~K}$. 
FOURIER P RUN 1076 BERYLLIUM $0.805 \mathrm{~K} \quad 273960001$
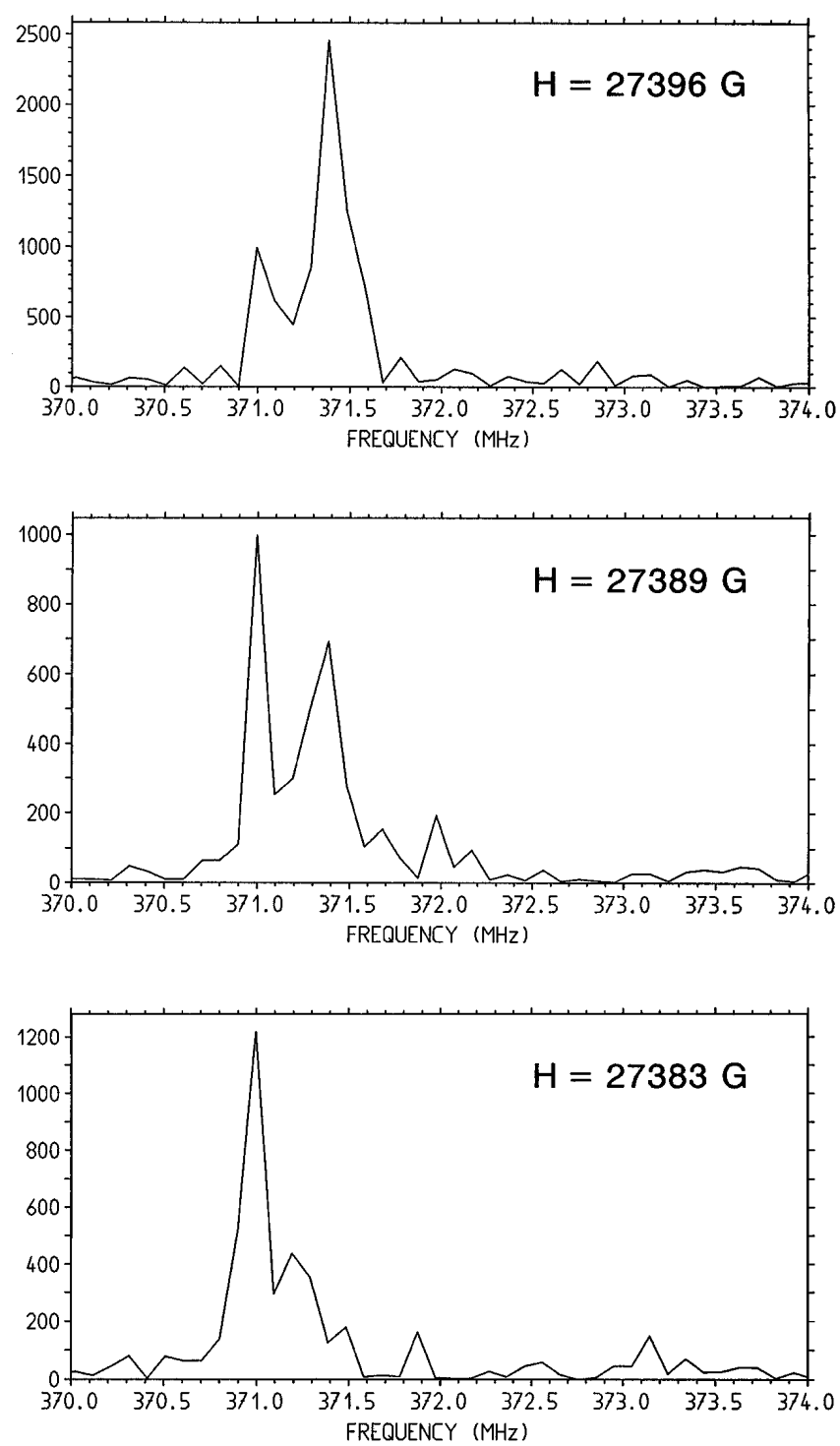

FIG. 2. Raw frequency spectrum data showing the split $\mu \mathrm{SR}$ line in the central peak region of Fig. 1. The applied field $H$ decreases downwards in the figure. Note the stable position of the doublet lines $(\Delta \nu=0.39 \mathrm{MHz})$ and the transfer of intensity from the higher frequency (paramagnetic domain) line to the lower one coming from the diamagnetic domains.

This evidence is detailed only in a quantitative way by plotting the result of a systematic fit of data in Fig. 3, where the frequency or frequencies in the $\mu$ SR spectra are plotted, together with the relative intensities of the lines. Since $\nu=\left(\gamma_{\mu} / 2 \pi\right) B$, the ordinates are just the $B$ values in the sample in $\mathrm{MHz}$ units.

The difference in induction between the domains is $\Delta B=28.8 \pm 1.4 \mathrm{G}$ at $H_{0}=2.740 \mathrm{~T}$. The measurement was repeated within a dHvA period around the $\approx 1000 \mathrm{G}$ lower field $H_{0}=2.644 \mathrm{~T}$, and the formation of domains was observed in the same way, with an even larger splitting $\Delta B \approx 40 \mathrm{G}$ in this field range. With increasing

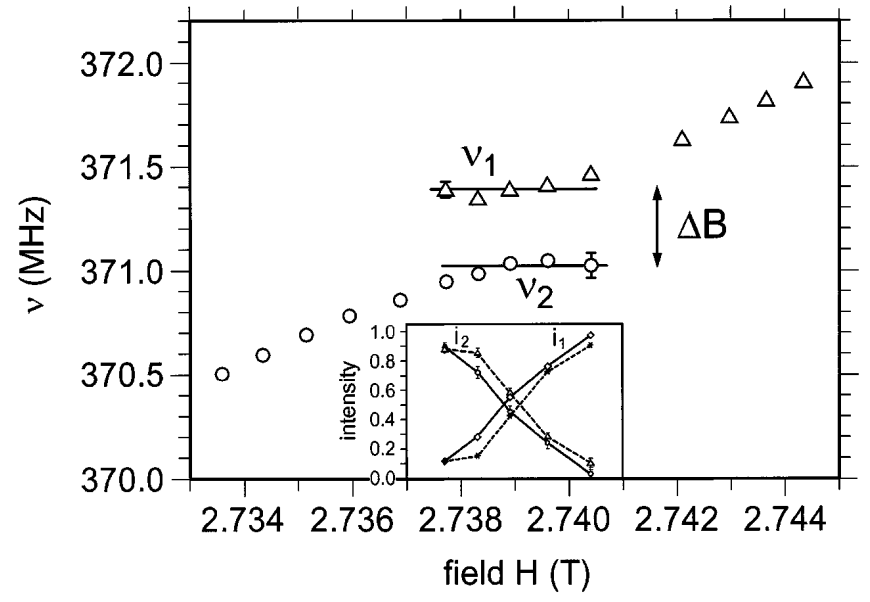

FIG. 3. Analysis of the central peak region of Fig. 1. The splitting of the frequency $\Delta \nu=0.39 \pm 0.02 \mathrm{MHz}$ for $27378<H<27405 \mathrm{G}$ corresponds to the difference in induction of $\Delta B=28.8 \pm 1.4 \mathrm{G}$ in the domains. The intensities $i_{1}, i_{2}$ (see inset) of the doublet lines show the relative volumes of paramagnetic and diamagnetic regions at the actual value of $H\left[i_{k}=I_{0, k} /\left(I_{0,1}+I_{0,2}\right)\right.$, and the solid and dashed curves correspond to the two detectors perpendicular to the muon beam].

the temperature from $0.8 \mathrm{~K}$, the splitting $\Delta B$ begins to decrease at $\approx 1.5 \mathrm{~K}$, it is reduced to about $9 \mathrm{G}$ at $T \approx 3.5 \mathrm{~K}$, and cannot be well resolved above this temperature. This is consistent with an earlier result on a similarly oriented single crystal sample [6] where, at the somewhat lower field $H_{0}=2.1 \mathrm{~T}$, Eq. (1) was seen to be no longer fulfilled above $\approx 3 \mathrm{~K}$.

In conclusion, the recurrent $\mathrm{dHvA}$ phase in beryllium was clearly demonstrated by the $\mu \mathrm{SR}$ spectra; the difference $\Delta B$ for the diamagnetic and paramagnetic domains was determined at the applied fields near $H_{0}=2.64$ and $2.74 \mathrm{~T}$. The ratio $\Delta B / \Delta H$ was found to be $\approx 0.37$ at $H_{0}=2.74 \mathrm{~T}$, it increases in this field range with lowering $H_{0}$. Due to the absence of quadrupolar linewidth and the conveniently large penetration length, $\mu$ SR spectroscopy seems to be a priori the best, and for $I>1 / 2$ actually the only experimental method suited for the study of the diamagnetic domain phase. This is the case of $\mathrm{Be}$, where a special advantage is the not too high external field at which the domains are formed. A detailed analysis of the domain structure, like the variation of width and wall thickness with $H_{0}$, as well as of the nature of the phase transition, is in progress.

The authors are indebted to F. Lykov (Kurchatov Institute) for preliminary thermopower measurements on the sample.

[1] J. H. Condon, Phys. Rev. 145, 526 (1966).

[2] D. Shoenberg, Magnetic Oscillations in Metals (Cambridge University, Cambridge, England, 1984).

[3] A. B. Pippard, Proc. Phys. Soc. London Sect. A 272, 192 (1963). 
[4] J. H. Condon and R. E. Walstedt, Phys. Rev. Lett. 21, 612 (1968).

[5] W.A. Read and J.H. Condon, Phys. Rev. B 1, 3504 (1970).

[6] L. R. Testardi and J.H. Condon, Phys. Rev. B 1, 3928 (1970).
[7] V. S. Egorov, Sov. Phys. JETP 45, 1161 (1977); Sov. Phys. Solid State 30, 1253 (1988).

[8] Yu. M. Belousov and V. P. Smilga, Sov. Phys. Solid State 21, 1417 (1979).

[9] A. Schenck, Muon Spin Rotation Spectroscopy (Hilger, Bristol, 1986). 